\title{
Let Disorder Dictate Topology
}

\author{
By varying the amount of disorder in a photonic crystal, researchers can \\ control several topological features of the crystal.
}

\section{By Matteo Rini}

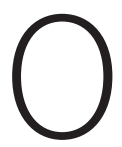
ne of the boons of topological insulators is that their conducting edge states are protected from disorder in the material's structure. Turn up the disorder too much, however, and these topological features are usually lost. In 2018, experiments demonstrated the opposite behavior: in so-called topological Anderson insulators (TAls), the addition of disorder can generate protected edge states, turning a trivial insulator into a topological one. Now, a team led by Baile Zhang of Nanyang Technological University in Singapore reports a new type of TAl realized in a photonic crystal [1]. Their experiments show that the crystal exhibits a wealth of topological phenomena that hadn't been seen previously.

The 2018 experiments, which used cold atoms and optical waveguides, provided the smoking gun of TAl behavior but could not test many predicted TAI features. Zhang and colleagues have engineered a setup that can more systematically test how disorder affects topology. Their system is a photonic crystal-a lattice of triangular nanopillars-in which they add the desired degree of disorder by randomly rotating the pillars.

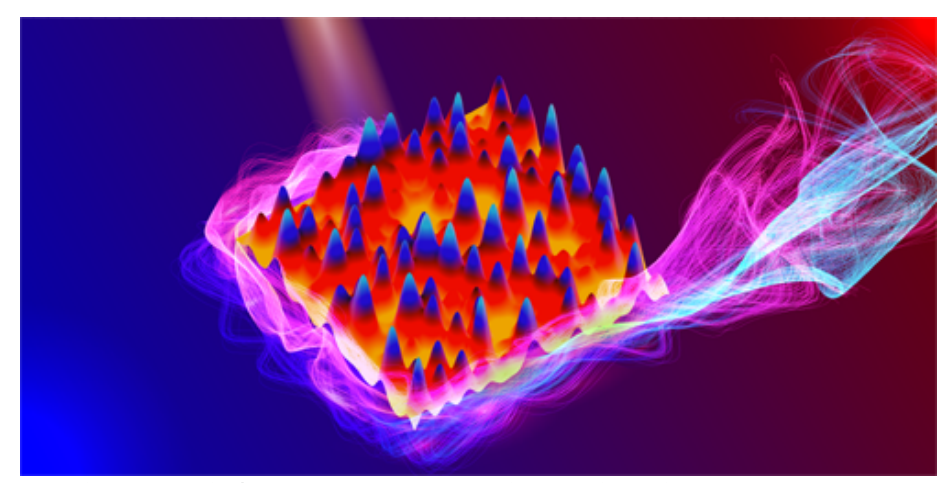

Credit: G.-G. Liu/Nanyang Technological University
The results show that, as in previous demonstrations, sufficiently large disorder makes the system topological-photons can't travel in the bulk but move freely at the crystal's edges. But the team found other, previously unseen, topological features. For instance, photons in edge states can bypass large defects and obstacles without scattering. The team also studied more complicated structures formed by neighboring domains with different disorder strengths. They show that the topological states can be routed to flow along the interfaces between the different domains-a behavior that could be useful in developing advanced nanophotonic circuits.

Matteo Rini is the Deputy Editor of Physics.

\section{REFERENCES}

1. G. Liu et al., "Topological Anderson insulator in disordered photonic crystals," Phys. Rev. Lett. 125, 133603 (2020). 